\title{
1 Similarities and differences of face and body perception in 2 the dog (Canis familiaris) and human brain
}

3 Magdalena Boch ${ }^{1,2^{*}}$, Isabella C. Wagner ${ }^{1}$, Sabrina Karl ${ }^{3}$, Ludwig Huber ${ }^{3+}$ and Claus

4 Lamm $^{1,4+}$

5 'Social, Cognitive and Affective Neuroscience (SCAN) Unit, Department of Cognition, Emotion, and

6 Methods in Psychology, Faculty of Psychology, University of Vienna, Vienna, Austria

$7 \quad$ 2Department of Cognitive Biology, Faculty of Life Sciences, University of Vienna, Vienna, Austria

$8{ }^{3}$ Comparative Cognition, Messerli Research Institute, University of Veterinary Medicine Vienna,

9 Medical University of Vienna and University of Vienna, Vienna, Austria

$10{ }^{4}$ Vienna Cognitive Science Hub, University of Vienna, Austria

11

$12+$ These authors share senior authorship.

13

$14{ }^{*}$ Corresponding author:

15 Email: magdalena.boch@univie.ac.at 
Neural bases of face and body perception in dogs and humans

16

17

18

19

20

21

22

23

24

25

26

27

28

29

30

31

32

33

34

\section{Abstract}

Accurately recognizing other individuals is fundamental for successful social interactions. While the neural underpinnings of this skill have been studied extensively in humans, less is known about the evolutionary origins of the brain areas specialized for recognising faces or bodies. Studying dogs (Canis familiaris), a non-primate species with the ability to perceive faces and bodies similarly to humans, promises insights into how visuo-social perception has evolved in mammals. We investigated the neural correlates of face and body perception in dogs $(N=15)$ and humans $(N=$ 40) using functional MRI. Combining uni- and multivariate analysis approaches, we identified activation levels and patterns that suggested potentially homologous occipito-temporal brain regions in both species responding to faces and bodies compared to inanimate objects. Crucially, only human brain regions showed activation differences between faces and bodies and partly responded more strongly to humans compared to dogs. Moreover, only dogs represented both faces and dog bodies in olfactory regions. Overall, these findings advance our understanding of the similarities and differences in brain function between two phylogenetically distant mammal species. This likely reflects differences in the perceptual systems these species rely on to recognize others, and provides important insights into the foundations of social cognition and behaviour. 
Neural bases of face and body perception in dogs and humans

\section{Introduction}

The ability to detect and recognize another individual is a socio-cognitive skill crucial for successful social interactions and survival. The face and body of another human or non-human animal convey a wealth of social information beyond their identity, such as when facilitating the correct categorization of another individual's emotional state [1-7]. Consequently, accurate perception of faces and bodies is an important building block for social perception.

Therefore, the neural underpinnings of face and body perception have been studied extensively in humans. Decades of research revealed a predominant role of the occipito-temporal cortex for the perception of others with distinct, but adjacent regions specialized for face and body perception as part of the so-called ventral visual pathway [8-17]. While the ventrolateral visual cortex responds to animate compared to inanimate stimuli more generally [18-20], the sensitivity in the occipital face and extrastriate body area (lateral occipital cortex), as well as the fusiform face and fusiform body area (inferior temporal cortex) appear increased for faces or bodies [10-13,1517,21-28]. Regarding the recognition of another individual's species-identity, previous research suggests a preference for human faces compared to faces of other species (such as dogs or horses) [29,30], potentially reflecting increased salience of conspecifics over heterospecifics.

The evolutionary history of the neural underpinnings of face and body perception has been mainly investigated by comparative research in non-human primates [21$24,28,31$ ], and this has revealed a homologous visual pathway [e.g. 8,32 for reviews]. However, apart from primate species, the neural bases of this socio-cognitive ability and its potential origin in the mammalian brain remain largely unstudied. Dogs' (Canis familiaris) ability to perceive and understand humans and conspecifics has already been well-investigated using behavioural and eye-tracking measures and yielded 
numerous comparable skills between dogs and humans. For example, dogs can not humans and conspecifics [7,35,36]; and they demonstrate complex behaviours such as visual perspective taking $[37,38]$. Thus, dogs constitute an excellent model to study the evolution of visuo-social perception in mammals [39].

While first non-invasive canine neuroimaging studies [29,40-43] suggested an involvement of the temporal cortex in face perception, they triggered a debate on whether the occipito-temporal specialization for face perception in dogs matches that of humans [29,40-43]. Previous work reported increased activation for faces compared to objects $[41,43]$, scenes [41], or a mono-coloured surface [40], but not compared to other low-level visual controls (i.e., scrambled images $[40,41]$ or the back of the head [29]). Some studies lacked non-facial controls entirely [42]. Studies additionally investigating responses to con- and heterospecifics were inconclusive ranging from reports of increased responsiveness to dog stimuli in general (i.e., face and back of the head) but no specialization for faces [29], over separate brain regions specialized for human or dog faces [42], to no species- but general face-preference [41]. Further, no previous work investigated the neural bases of body perception. This is a major limitation, as bodies play an important role for social perception in general, but in particular for dogs - as indicated e.g., by dogs' high responsiveness to emotional or ostensive-referential bodily cues, such as tail-wagging [44] or human gestures [4446]. It is particularly noteworthy in this respect, that dogs even outperform humans' closest living relatives, chimpanzees, in utilizing human ostensive cues [47]. general animacy perception and are inconclusive regarding the influence of the other individual's species-identity. The present comparative neuroimaging study aimed to resolve this debate. We performed the, to our knowledge, first systematic comparison 
Neural bases of face and body perception in dogs and humans

87 of the neural bases of face, body and inanimate object perception in dogs and humans,

88 in order to gain insights into how social perception has evolved in two phylogenetically

89 distant mammalian species. [39,48].

\section{Results}

Fifteen awake and unrestrained pet dogs (Figure 1A) and forty human

92 participants underwent functional magnetic resonance imaging (MRI) while viewing

93 images of human and dog bodies, faces, inanimate objects (e.g., different toys), and

94 grid-scrambled versions of these images serving as visual controls (Figure 1B). Over

95 the course of two 5 min runs, participants saw 180 different images presented in a

96 block design interspersed with an implicit visual baseline. We analysed the dog fMRI

97 data with a newly developed analysis protocol shown to significantly improve neural

98 signal detection power [49]. All statistical tests were corrected for multiple comparisons

99 (see fMRI data analysis, Figures 1-3, Supplementary file 1: Tables S1-S5). 
bioRxiv preprint doi: https://doi.org/10.1101/2021.08.17.456623; this version posted September 20, 2021. The copyright holder for this preprint (which was not certified by peer review) is the author/funder, who has granted bioRxiv a license to display the preprint in perpetuity. It is made available under aCC-BY-NC-ND 4.0 International license.

Neural bases of face and body perception in dogs and humans

A Set-up dog participants

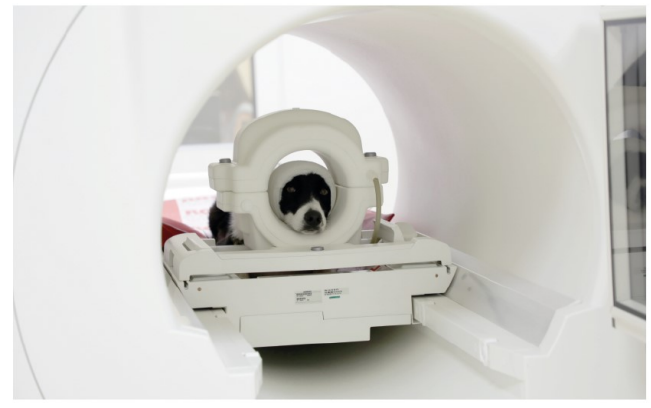

B Image categories

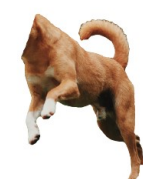

bodies dogs

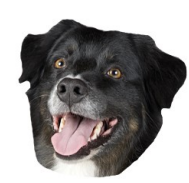

faces dogs

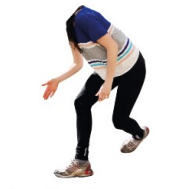

bodies humans

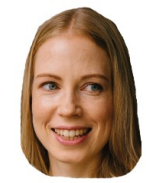

faces humans
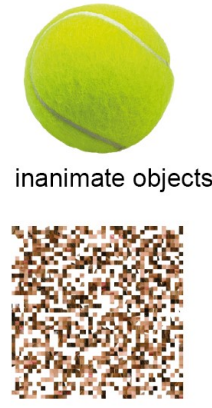

scrambled

C Task-responsive voxels (localizer data set: all stimuli $>$ baseline)
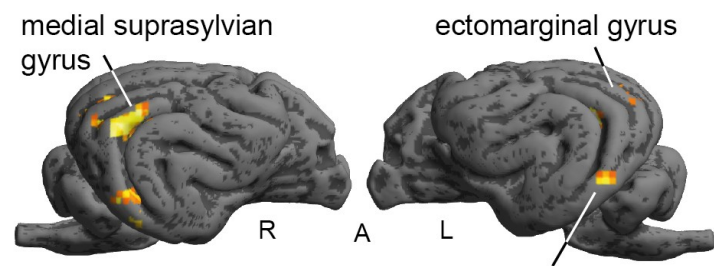

caudal suprasylvian gyrus

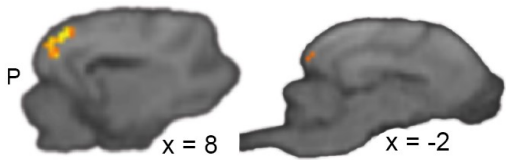

R marginal, splenial, L occipital gyrus

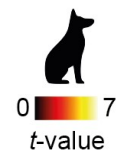

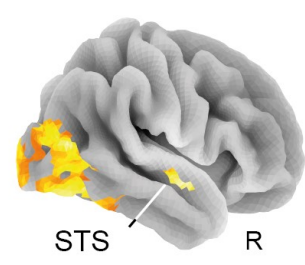
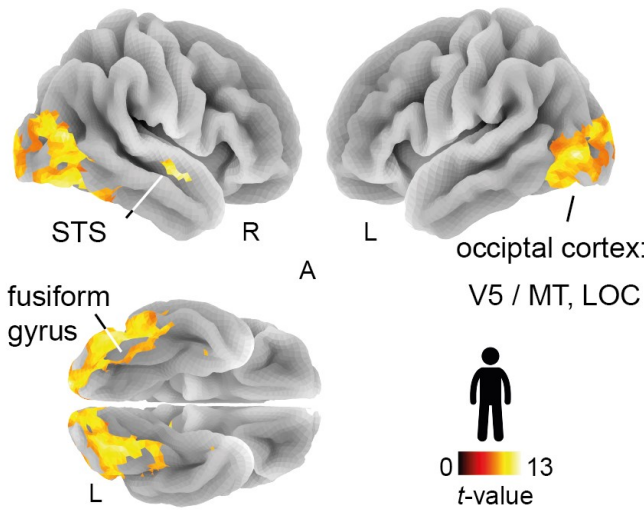

V5 / MT, LOC

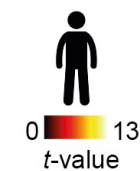

Figure 1. Overview of the comparative experimental approach to study the neural bases of face and body perception in dogs and humans. (A) We obtained all imaging data using a 3T Siemens Skyra MR-system, equipped with a 15-channel human knee coil to scan the dog participants, and using a 32-channel human head coil for the human participants (not depicted). Dogs received extensive training [50] to stay motionless without restraints or sedation and could leave the MR scanner at any time via a custom-made ramp positioned at the scanner bed. All (dog and human) participants wore earplugs for noise protection and dog participants had an additional head bandage to secure optimal positioning of the earplugs throughout the scan session. (B) All participants viewed images of human and dog bodies, human and dog faces, inanimate objects (e.g., toys) or gridscrambled versions of these images presented in a block design. (C) We split the data sets and used data from the first task run to compare activation for visual stimulation (all stimuli) to the visual baseline (i.e., the localizer data set). In both participant samples, we observed activation in the occipito-temporal cortices. These task-responsive voxels then served as a search space to determine individual functional regions-of-interest (fROIs), additionally constrained by bilateral anatomical regions (see Figure 2A). Results are displayed at $p<0.05$, FWE-corrected at cluster-level using a cluster-defining threshold of $p<$ .001 (dogs, Supplementary File 1: Table S1), and $p<0.05$ whole-brain FWE-corrected (humans; Supplementary File 1: Table S1) Results for the dogs are overlaid onto a canine breed-averaged surface render [51], and onto the FreeSurfer "FsAverage" surface for humans (https://surfer.nmr.mgh.harvard.edu/fswiki). Anatomical nomenclature for all figures refers to the dog brain atlas from Czeibert and colleagues [52] and to the human brain atlas from the Laboratory for Neuro Imaging (LONI) Brain Atlas [53] (LPBA40; http://www.loni.usc.edu/atlases/). R, right; L, left; A, anterior; P, posterior; STS, superior temporal sulcus; MT, middle temporal visual area (V5); LOC, lateral occipital cortex.

\subsection{Mass-univariate analysis: Face- or body-sensitive areas in the}

\section{occipito-temporal cortices of dogs and humans}

To start out, we investigated whether dogs and humans have specialized cortical 
Neural bases of face and body perception in dogs and humans

106

107

108

109

110

111

112

113

of-interest analysis approach (fROI,[54-56]). We split the data into two orthogonal data sets, (1) a localizer data set (first task run) to define potential face- or body-sensitive areas in visual-responsive brain regions (Figure 1C, 2A) and (2) a test data set (second task run) to extract activation levels from these regions and to test potential category sensitivity. We chose this approach for two reasons. First, we aimed at directly testing the potential category-sensitivity, quantified by increased activation levels. Second, our goal was to account for differences in the location of activation peaks between individuals as reported in previous dog imaging studies $[41,43]$.

\subsubsection{Functional regions-of-interest (fROls)}

For each participant, we defined bilateral fROIs within constrained search spaces to preserve spatial information (i.e., the rough anatomical location of activation peaks). For the human participants, we used previously reported anatomical regions known to be engaged in face and body perception [57]: the extrastriate body area, fusiform body area, occipital face area and fusiform face area (Figure 2A). For the dog participants, we could not build on previous research due to lack of shared template space and availability of data. Therefore, we first compared activation levels associated with the visual presentation of all stimuli compared to implicit baseline (i.e., white cross presented on grey background) using the localizer data set. This revealed taskresponsive activation within the occipital-, splenial-, ectomarginal-, caudal-, medial suprasylvian- and marginal gyri, partially overlapping with results from previous studies investigating face perception [29,40-43,58,59] (Figure 1C, Supplementary File 1: Table S1). We then used anatomical masks [52] of these regions as search spaces for the dog participants (Figure 2A). For comparison, we also report task-responsive voxels for the human participants, confirming involvement of the occipito-temporal cortex including the lateral occipital cortex and fusiform gyrus (Figure 1C, 
Neural bases of face and body perception in dogs and humans

131 Supplementary File 1: Table S1; see also Figure 4 for schematic figures of the dog 132 and human brain).

We then defined individual category-sensitive regions within each search space by selecting the top-10\% voxels with the strongest activation for bodies or faces compared to inanimate objects based on the individual data from each participant.

\subsubsection{Sensitivity for animate stimuli but sub-division in face- and body- sensitive regions exclusive to humans}

Next, we extracted the mean activation signal during viewing of faces, bodies,

inanimate objects and scrambled images (all compared to the implicit baseline) from

140 the individual fROls using the test data set. In dogs, we observed significantly

141 increased activation levels for faces and bodies compared to inanimate objects or

142 scrambled images in both face and body fROIs, located in the medial and caudal

143 suprasylvian- and ectomarginal gyrus. In the caudal suprasylvian face- and

144 ectomarginal body fROI, the increased activation for faces showed the same trend but

145 did not always reach significance. We did not find consistent evidence for a further

146 sub-division into face- and body-selective areas in the occipito-temporal cortex, as

147 there was no significant activation difference between faces and bodies in seven out

148 of eight fROls. We only observed significantly increased activation for bodies

149 compared to faces in the caudal suprasylvian face but not body fROI. The occipital and

150 splenial face and body fROls did not result in differential activation changes in

151 response to any of the stimulus categories (Supplementary File 1: Table S2).

152 Regarding the parietal cortex, the marginal gyrus appeared solely responsive to bodies

153 compared to all other conditions with no activation differences for faces compared to

154 inanimate objects or scrambled controls (Figure 2B). 
bioRxiv preprint doi: https://doi org/10.1101/2021.08.17.456623; this version posted September 20, 2021. The copyright holder for this preprint (which was not certified by peer review) is the author/funder, who has granted bioRxiv a license to display the preprint in perpetuity. It is made available under aCC-BY-NC-ND 4.0 International license.

Neural bases of face and body perception in dogs and humans

A Bilateral anatomical search spaces to create individual functional regions-of-interest (fROls)

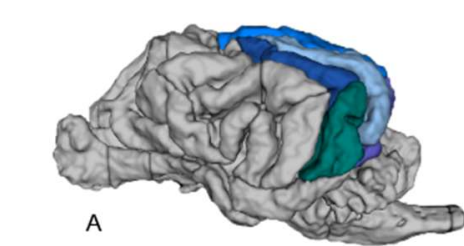

left lateral view

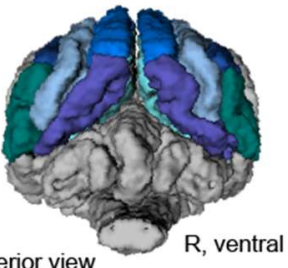

posterior view

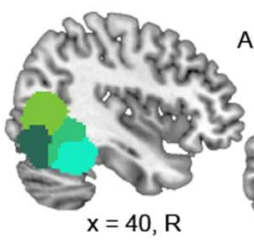

$x=40, R$
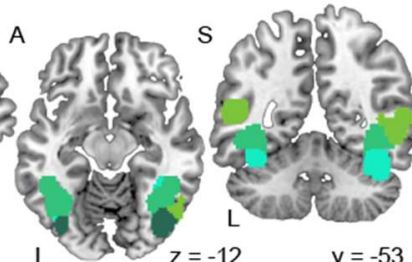

$y=-53$

medial suprasylvian gyrus

caudal suprasylvian gyrus

marginal gyrus

splenial gyrus

ectomarginal gyrus

occipital gyrus

extrastriate body area
fusiform body area

occipital face area

fusiform face area

B fROI analysis: mean activation extracted from independent test data set
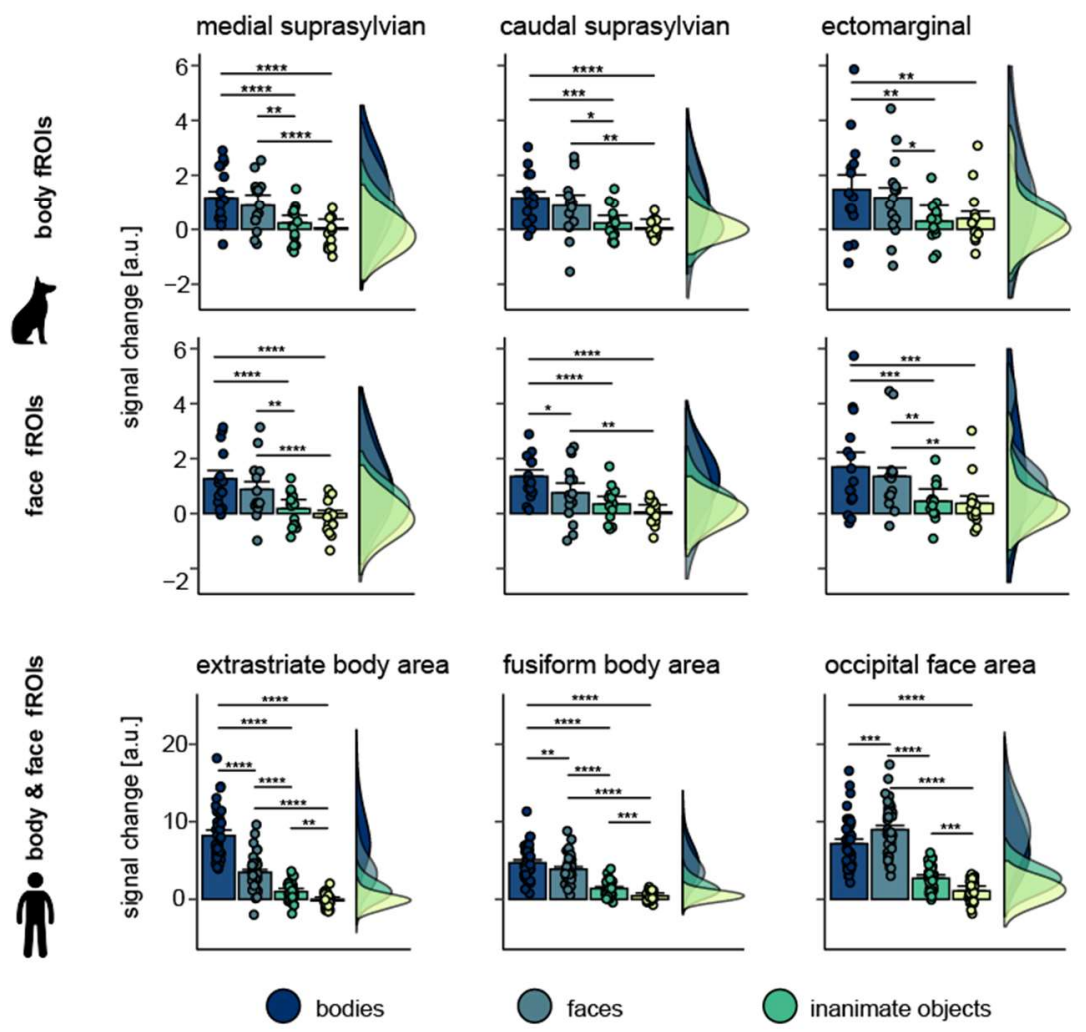

marginal
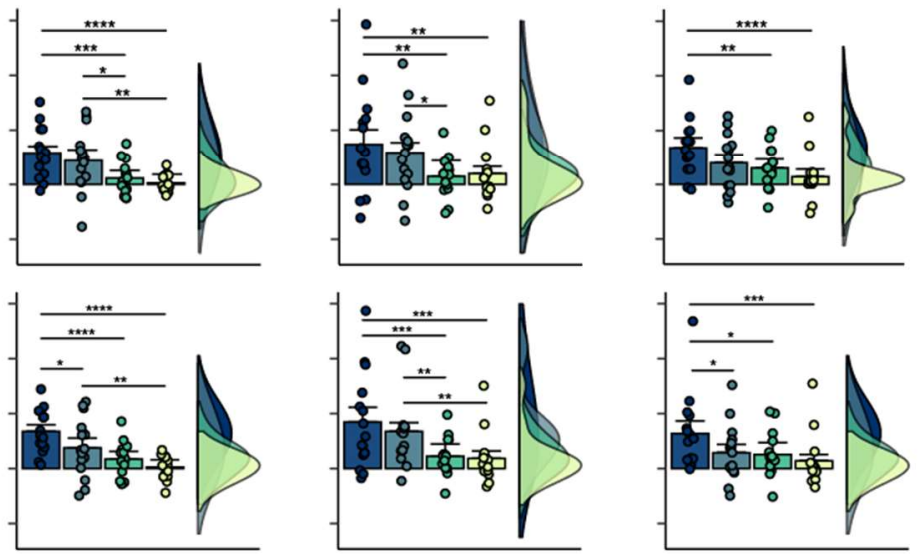

fusiform body area

occipital face area
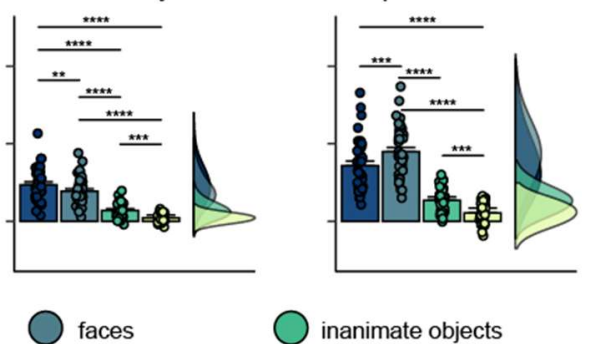

fusiform face area

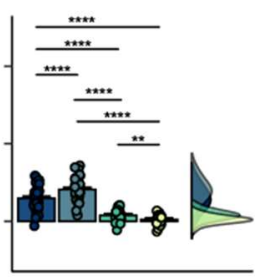

inanimate objects

scrambled

Figure 2. Strongest activation levels for animate stimuli in both species, but differences between faces and bodies exclusive in humans (A) Based on the localizer data set, we defined individual category-specific regions-of-interest (functional region-of-interest approach, fROI) within multiple anatomically constrained search spaces using the contrasts bodies > inanimate objects (body fROIs) and faces > inanimate objects (face fROls). (B) From these individual fROIs, we then extracted activation estimates during the viewing of faces, bodies, inanimate objects and scrambled images (all compared to the implicit baseline) using the data from the independent test data set to test potential face- or body-sensitivity. (B) In dogs, we observed increased activation levels for faces and bodies compared to inanimate objects or scrambled images in three occipito-temporal regions (medial and caudal suprasylvian, ectomarginal gyrus) in face and body fROls. However, no difference between faces and bodies except in the caudal suprasylvian face fROI. The face and body fROIs located in the occipito-parietal marginal gyri resulted in increased activation levels primarily for bodies. In humans, similar to dogs, we observed the strongest activation levels for faces and bodies in all fROls. In contrast to the dog data, the human data analysis consistently resulted in a significant activation change for bodies compared to faces in body fROIs and vice versa (Supplementary File 1: Table S2). Planned comparisons were corrected for multiple comparisons using Tukey method. ${ }^{*} p<.05,{ }^{* *} p<.01,{ }^{* * *} p<.001,{ }^{* * *} p<$ .0001 , error bars represent the standard error of the mean; a.u., arbitrary units. Atlas maps and search spaces refer to the dog brain atlas from Czeibert and colleagues [52] and human parcels were retrieved from previous work [57]. A, anterior; P, posterior; S, superior; $L$, left; $R$, right. 
bioRxiv preprint doi: https://doi org/10.1101/2021.08.17.456623; this version posted September 20, 2021. The copyright holder for this preprint (which was not certified by peer review) is the author/funder, who has granted bioRxiv a license to display the preprint in perpetuity. It is made available under aCC-BY-NC-ND 4.0 International license.

Neural bases of face and body perception in dogs and humans

158

159

160

161

162

163

164

165

166

167

168

169

170

171

172

173

174

175

176

177

178

179

180

181

182

183

body areas and for faces in the occipital and fusiform face areas (Figure 2B,

Supplementary File 1: Table S2). In summary, we found multiple occipito-temporal regions in the dog and human cortices responsive to animate stimuli, but detected a further sub-division into face- and body-sensitive regions exclusively in the human brain (see also Figure 4 for a summary of the results).

\subsubsection{Conspecifics preference in human extrastriate body and fusiform face area}

Next, we investigated whether processing of faces or bodies differed when participants saw images of con- compared to heterospecifics (Supplementary File 1:

Table S3). In humans, this revealed increased activation for human (mean: 4.46, SD $=1.83$ ) compared to dog bodies (mean: $3.76, S D=1.66$ ) in the extrastriate body area $(t(39)=3.14, p=0.003$, mean Diff: $0.7,95 \% \mathrm{Cl}[0.25,1.15])$, as well as a general activation increase for human (mean: 1.89, $S D=0.87$ ) compared to dog (mean: 1.64, $S D=0.81)$ stimuli in the fusiform face area $(t(79)=3.71, p<0.0001$, mean Diff: 0.25 , 95\% Clmeandiff $[0.16,0.38])$. For the dogs, we did not find a difference between the perception of dog and human stimuli.

\subsection{Multivariate pattern analysis of animacy perception}

Despite the overall activation (measured using mass-univariate analyses) to stimulus categories may not differ, we reasoned that more fine-grained analyses of multivariate activation patterns could reveal distinctions [60]. Therefore, we investigated the neural representations of faces and bodies in dogs and humans and their potential correspondence using whole-brain representational similarity analyses (RSA). We moved a 4 and $8 \mathrm{~mm}$ searchlight across the whole dog and human brain, respectively, in order to determine individual pattern similarity maps between all trials of each stimulus category [61-63] (Figure 3A). On the group-level, we then conducted paired $t$-tests to compare the pattern similarities maps of (1) animate vs. inanimate 
Neural bases of face and body perception in dogs and humans

184 stimuli, (2) bodies vs. inanimate objects, (3) faces vs. inanimate objects, (4) faces vs. bodies, and $(5,6)$ within the face and body categories: images of conspecifics vs. heterospecifics (i.e., dog faces vs. human faces; dog bodies vs. human bodies).

Results revealed increased (within-category) pattern similarity for animate compared to inanimate stimuli in the occipito-temporal cortex of dogs (i.e., caudal suprasylvian and splenial gyrus) and humans (i.e., middle occipital gyrus) overlapping with the identified fROIs. In human participants, we additionally observed increased similarity in the cerebellum and fronto-parietal regions (Supplementary File 1: Tables

S4-S5). In brief, this indicates distinct neural representations of animate compared to inanimate stimuli in higher-order visual regions of both species.

\subsubsection{Similar and divergent neural representations of bodies and faces}

Moving on to neural representations for bodies and faces, for bodies we observed

increased pattern similarity compared to inanimate objects in higher-order visual areas

in the occipito-temporal cortex, partially overlapping with the identified fROIs, and the

cerebellum in both species (Figure 3B). Within the same regions (and again, in both species), we also observed distinct activation patterns for conspecific compared to heterospecific bodies. Furthermore, when the dogs viewed dog bodies, results revealed increased similarity in limbic structures and regions associated with olfaction

(Figure 3C). In humans, we observed increased similarity for human compared to dog bodies in fronto-parietal regions associated with action perception [64-66] (Figure 3C). Observing faces compared to inanimate objects resulted in significantly increased pattern similarity in occipito-temporal cortices of both species and again in dog olfactory structures (Figure 3D). Comparing similarity patterns for faces vs. 207 bodies, we only found significant differences for the human participants (see 208 Supplemental file 1: Tables $\mathbf{S 4 - 5}$ for detailed results and Figure 4 for a schematic 209 summary of the main results). 
bioRxiv preprint doi: https://doi.org/10.1101/2021.08.17.456623; this version posted September 20, 2021. The copyright holder for this preprint (which was not certified by peer review) is the author/funder, who has granted bioRxiv a license to display the preprint in perpetuity. It is made available under aCC-BY-NC-ND 4.0 International license.

Neural bases of face and body perception in dogs and humans

A Contrasts representational similarity analysis (RSA): within condition similarities
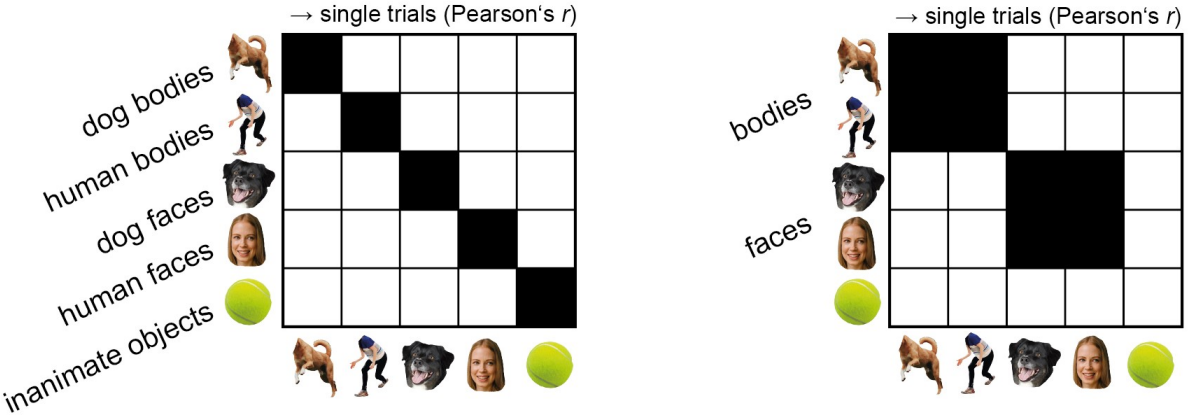

B Paired $t$-test RSA: bodies > inanimate objects
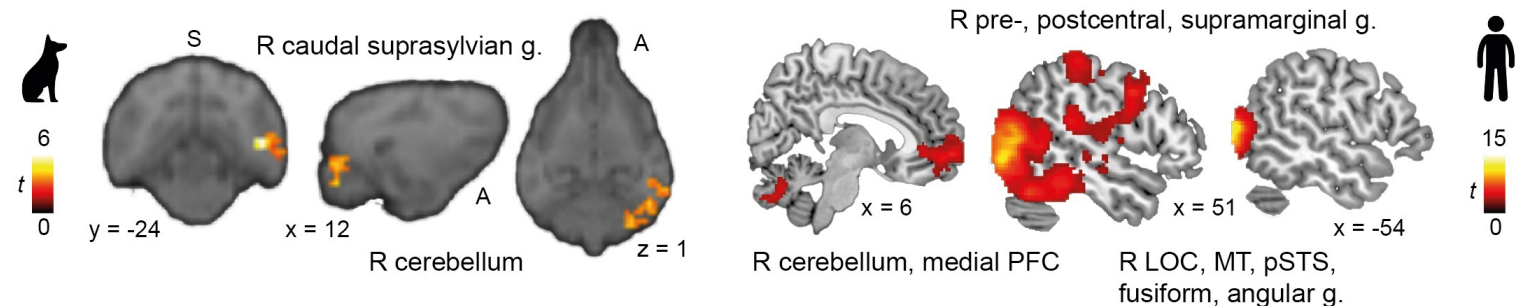

C Paired $t$-test RSA: conspecific bodies $>$ heterospecific bodies

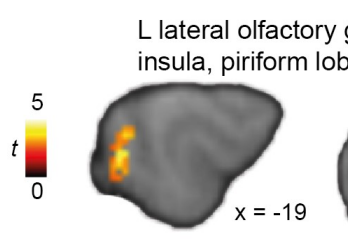

L caudal suprasylv. g
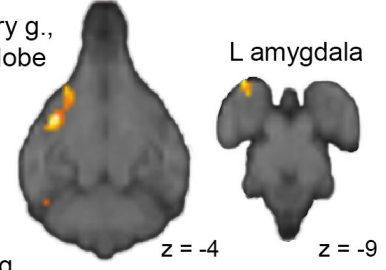

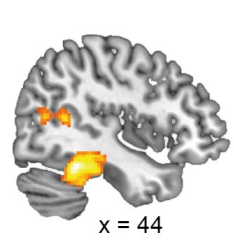

$\mathrm{R}$ fusiform, angular $\mathrm{g}$.

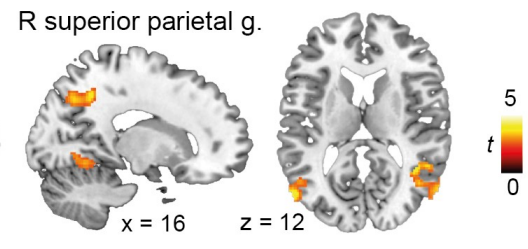

R lingual g. bilateral occipital $\mathrm{g}$.

D Paired $t$-test RSA: faces > inanimate objects
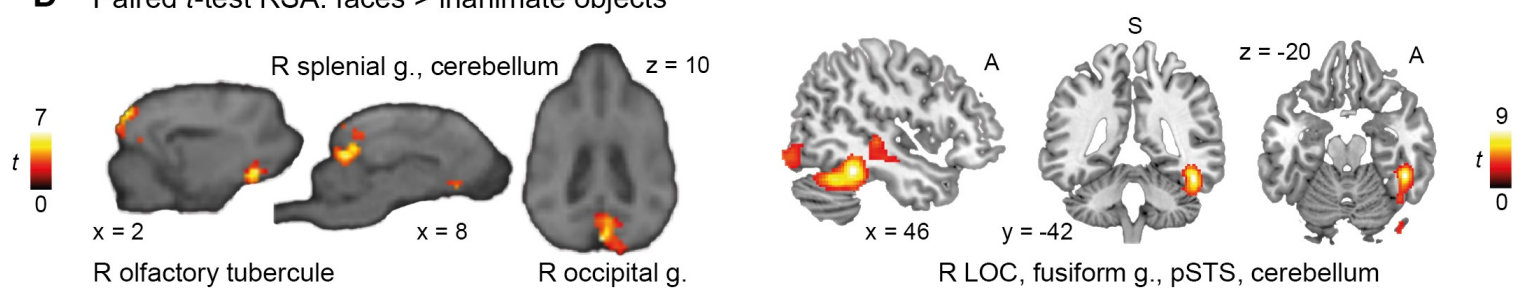

Figure 3. Whole-brain representational similarity analysis (RSA) revealed similar and divergent neural patterns of activation for animate vs. inanimate stimuli but differences between faces and bodies again exclusive in human. (A) We moved a $4 / 8 \mathrm{~mm}$ (dog/human) spherical searchlight across the whole brain to quantify the neural patterns underlying stimuli categories. Pattern similarity matrices were computed across the respective quadrants marked in black. (B,D) In both species, we observed increased pattern similarity for bodies or faces compared to inanimate stimuli in higher-order visual areas in the occipito-temporal cortex and the cerebellum. (C,D) In dogs we additionally detected distinct neural patterns of activation for faces and conspecific (= dog) bodies in olfactory and limbic regions. In humans, we observed an increased pattern similarity for (conspecific) bodies in fronto-parietal regions associated with action perception [64-66]. All results are displayed at $p<0.05$, FWE-corrected at cluster-level, and using a cluster-defining threshold of $p<.005$ for the dog and $p<.001$ for the human data (Supplementary file 1: Tables S4-5). Anatomical locations are shown in panel B for the dog and in D for the human data: superior (S), anterior (A); all sagittal, coronal and axial planes displayed have the same orientation. Coordinates refer to a canine-breed averaged template [51] or to MNI space for the human data. Person's $r$, correlation coefficient; $t, t$-value; g., gyrus; PFC, prefrontal cortex LOC, lateral occipital cortex; MT, middle temporal visual area (V5); pSTS, posterior superior temporal sulcus; $R$, right; L, left. 
Neural bases of face and body perception in dogs and humans

\section{Discussion}

Our findings revealed that the occipito-temporal cortex has a prominent role in

214 the perception of animate entities in both dogs and humans. This suggests that the

neural processing of animate vs. inanimate stimuli across mammalian brains may either have an old evolutionary origin in the common ancestors of canines and primates

217 or is the result of convergent evolution [48]. However, within these regions, only

218 humans displayed an additional sub-division into distinct face- and body-sensitive brain regions. Moreover, only dogs showed distinct responses to human and dog faces as well as dog bodies in olfactory areas (see Figure 4 for schematic summary of the results). These findings suggest a divergent evolution of the neural bases of face and body perception (or processing) in dogs and humans. The differential engagement of

223 visual and olfactory brain functions would fit particularly well with the differential 224 sensitivity and preferential use of these perceptual systems to infer social and contextual information.

Detecting biological agents is crucial for survival and social relationships. Hence,

227 the visual differentiation between animate vs. inanimate entities is an evolutionary

228 important category representation. Studies show that dogs target animate agents 229 when, for example, presented with images of wild animals, humans or dogs embedded 230 in natural landscapes [67] or of social interactions between humans and dogs [68].

231 Further, dividing stimuli into an animate vs. inanimate dimension is one of the first 232 visually salient categorizations formed by human infants [69]. In this way, animacy 233 representation provides the first building block for more complex visual categorizations 234 such as faces vs. bodies. Thus, considering its biological significance and the observed cross-species similarities in our study, animacy might constitute a general organizing principle across mammalian brains. 


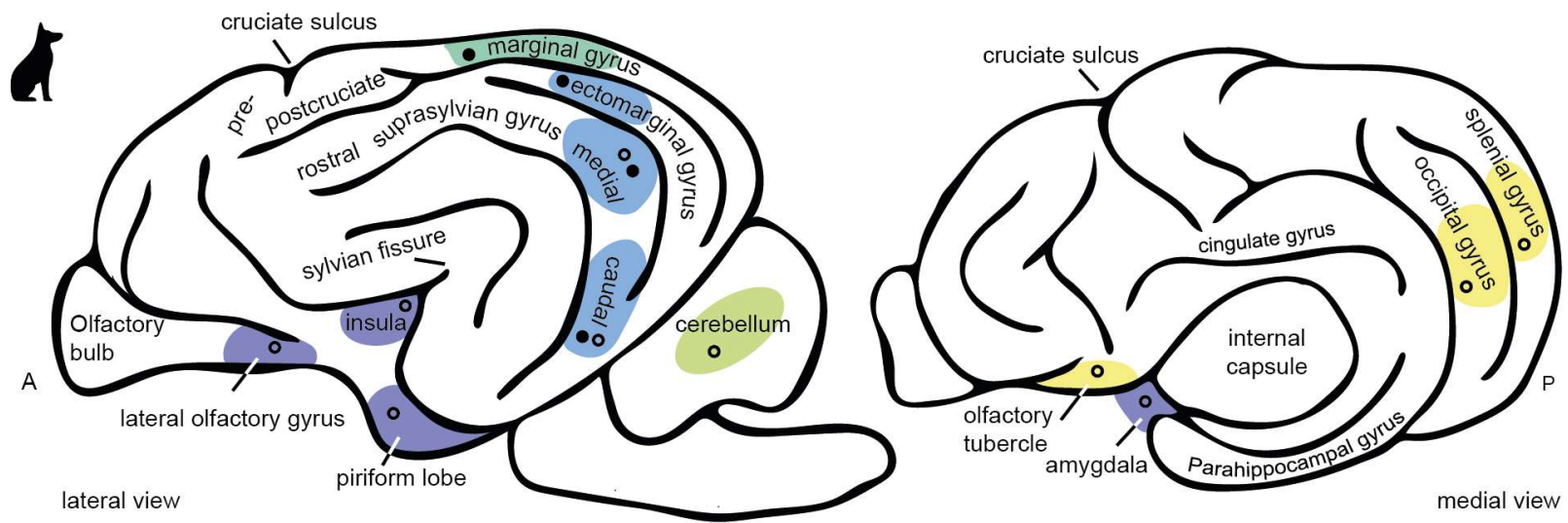

\section{(6) (3) is
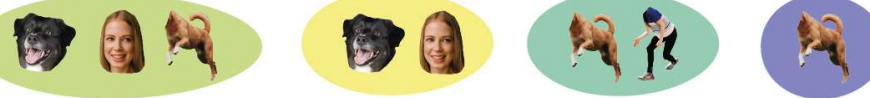
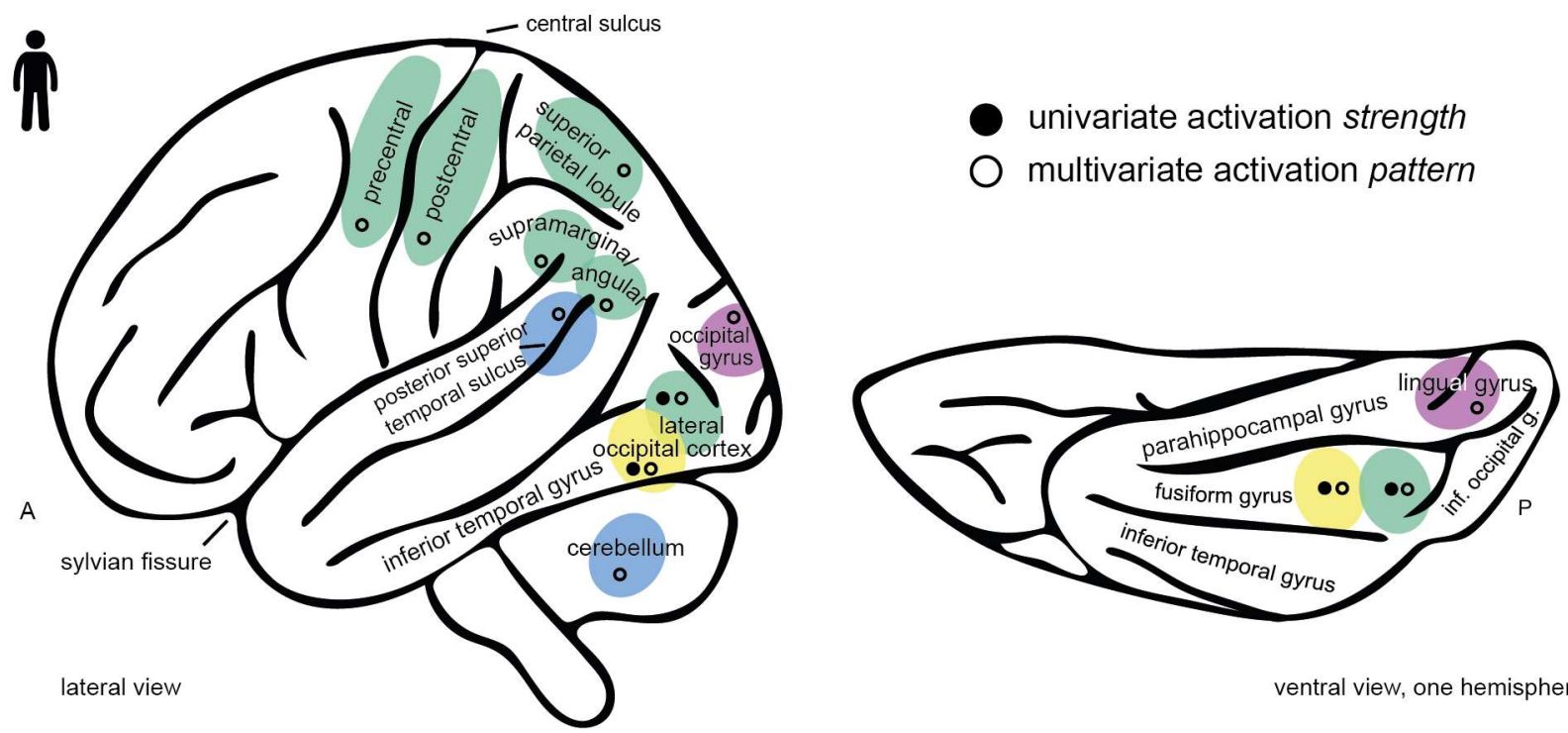

ventral view, one hemisphere

Figure 4. Summary of the main study findings. Results from the functional regions of interest (univariate activation strength) and representational similarity analysis (multivariate activation pattern) illustrating the results on schematic brain figures. For simplification, observed results are always summarized on one hemisphere; they do not mark the exact but the approximate anatomical location. already as puppies [76]. Our results do not contradict these findings, but suggest that

242 visual regions involved in the perception of faces are also involved in the perception of other body parts, and may thus encode animate stimuli. A recent dog neuroimaging 
Neural bases of face and body perception in dogs and humans

245 in the perception of complex social interactions, which would confirm their important 246 role for social perception.

$247 \quad$ While previous investigations of how dogs perceive bodies have mainly focused 248 on the decoding of human referential cues, we hope that our work will inspire more 249 research on how dogs perceive bodily social as well as emotional cues of con- as well as heterospecifics. The absence of a further sub-specialization for face vs. body perception in the occipito-temporal cortex of dogs might indeed indicate that dogs in comparison to humans focus more on whole body social cues rather than on specific sub-parts. This interpretation is notably well in line with a recent comparative eye254 tracking study [78].

Regarding species perception, our results indicate an increased salience for human compared to dog stimuli in humans, but no evidence for a conspecific257 preference in the occipito-temporal cortex of dogs. Thus, human and dog stimuli appear to be equally salient for dogs. This is in line with previous behavioural studies suggesting, for example, no significant difference in the perception of human or dog emotional facial [35], whole-body cues [78], or images of social interactions [68].

261 Further, two studies demonstrated that dogs, already as puppies, follow human gestural-communication and show an interest in human faces [76], which has not been observed in wolf puppies [79]. Additionally, half of the variation in these socio-cognitive 264 skills could be accounted for by genetic factors, suggesting that dogs' attention to humans might have been enhanced during domestication [75 for review of both studies]. Future studies investigating species-preferences in dogs might thus consider 267 adding further heterospecific stimuli of other familiar species, such as cats. In accordance with a potential divergent evolution of face and body 269 representations in dogs and humans, we also observed distinct neural representations 270 for faces (regardless of species) and conspecific (dog) bodies in dog olfactory regions. 
Neural bases of face and body perception in dogs and humans

271 Although unexpected, these results might reflect dogs' significantly higher olfactory

272 sensitivity and it's use to infer social and contextual information [54 for review].

273 However, the link between cognition and olfaction remains speculative since

274 behavioural studies using olfactory cues so far mainly explored dogs' odour and

275 disease detection abilities (e.g., [82-86] while dog imaging studies on olfaction focused

276 on reward-processing [87,88] or general sensory abilities [89-91]. However, a recent

277 behavioural study investigating dog cognition using olfactory cues showed first 278 evidence that dogs form representations of their owners when smelling their tracks 279 [92].

In humans, results revealed distinct activation patterns for bodies in fronto281 parietal regions that have been consistently associated with the perception of actions [64-66]. In dogs, we did not find activation in (pre-)motor cortices, but the increased neural sensitivity for bodies in the occipito-parietal marginal gyrus might be initial 284 evidence for an analogous visual pathway to encode actions. However, this is beyond our study's scope, and the precise neural underpinnings of action observation in dogs have not yet been investigated.

We have taken several steps to maximize neural signal sensitivity already when designing our study (e.g., by using a block design) and at the analysis stage (e.g., by using dog-tailored hemodynamic models increasing response detection power [49]).

290 Nevertheless, the extensive training required for dogs [50] resulted in different sample 291 sizes for the two species, and the more extensive prior work in humans [57] resulted 292 in more constrained search spaces, and likely higher spatial sensitivity for the human 293 data. Further, the majority $(80 \%)$ of our pet dogs were pure-bred herding dogs, which 294 prevented us from testing potential breed-specific differences [as in e.g. 90] and may 295 limit generalizability. 
Neural bases of face and body perception in dogs and humans

In conclusion, our study reveals novel evidence for similarities and differences of

297 animacy perception between two phylogenetically distant mammal species, advancing

298 our understanding of the foundations of social cognition and behaviour. Finally, we

299 provide insights into the differentially evolved sensory systems of dogs and humans

300 for the perception of faces and bodies.

3014 Materials and Methods

\subsection{Participants}

Fifteen pet dogs (Canis familiaris; 11 females, age range: 4-11 years, mean age:

7.8 years) consisting of 10 Border collies, 2 Australian shepherds, 1 Labrador retriever and 2 mixed-breed dogs and forty human participants (22 females, age range: 19-28 years, mean age: 23 years) participated in the present study.

For the dog sample size we aimed to collect data from a minimum of $N=12$ dogs during the data collection period from April 2019 to July 2020 which was the median sample size of task-based dog fMRI studies at the time of planning the study (the median sample size in 2021 is also $N=12$ ). For the human participants, we aimed for a sample size of $N=40$ participants, based on previous studies in our lab with similar task designs, and also being in line with a previous study [57] investigating the neural bases of face and body perception using a functional region-of-interest approach with a sample size of $N=35$ participants (reporting $F$-values above 40 for all repeated 315 measures analyses).

Prior to MRI scanning, all dogs underwent initial medical examination concerning

317 their eyesight and general health and received extensive training to habituate to the

$318 \mathrm{MRI}$ environment [50]. They were fully awake, able to exit the MRI scanner at any time 319 and equipped with noise protection (Figure 1A). All caregivers gave informed written 320 consent to their dogs' participation. Human participants were right handed, had normal 
Neural bases of face and body perception in dogs and humans

or corrected-to-normal vision, they reported no history of neurological or psychiatric

disease, nor phobia of dogs, fulfilled the standard inclusion criteria for functional MRI, and gave informed written consent to participate. The study complies with the ARRIVE guidelines [94]. Dog data collection was approved by the institutional ethics and animal welfare commission in accordance with Good Scientific Practice (GSP) guidelines and national legislation at the University of Veterinary Medicine Vienna (ETK-06/06/2017), based on a pilot study conducted at the University of Vienna. Human data collection was approved by the ethics committee of the University of Vienna (reference number: 00565) and performed in line with the latest revision of the Declaration of Helsinki 330 (2013).

\subsection{Task and procedure}

We employed a block design (duration: $12 \mathrm{~s}$ ) split in two 5 min runs where participants saw images of faces and bodies of dogs or humans, objects and an MR-compatible screen (32 inch) positioned at the end of the scanner bore. Crucially, we used the same task for dogs and humans. Human participants were instructed to watch the images presented on the MR screen and dogs were trained to attend to the MR screen (passive viewing paradigm). Each run contained three blocks per condition, block order was randomized but the same condition was never presented twice in a row. Between blocks, participants saw a visual baseline jittered between 3-7 s with a

341 white cross presented on grey background. Image composition for each block and 342 order within each block was randomized across participants to ensure effects are not 343 driven by specific blocks; each image was presented once. The task was implemented 344 using PsychoPy [95]. Overall motion and wakefulness were live monitored via the 345 camera of an eye-tracker (Eyelink 1000 Plus, SR Research, Ontario, Canada) 346 positioned below the MR-compatible screen. For the dogs, the trainer stayed within the 
Neural bases of face and body perception in dogs and humans

scanner room but out-of-sight throughout the scan session to monitor and handle the

348 dogs. Human participants saw both runs within a single scanner session with a short

349 break in-between. For the dogs, the number of attempted sessions varied depending

350 on how many repetitions they needed to complete one run without substantive motion

351 and with sufficient attentiveness (i.e., eyes open and gazing towards the centre of the screen); in-between task runs the dogs always were given a short break outside the MR scanner. Realignment parameters were evaluated after each scan session. If overall motion exceeded $4 \mathrm{~mm}$ in any of the three translation directions, the dog was re-invited to repeat the run in a subsequent session and sessions were scheduled at least one week apart. On average, dogs needed three sessions to complete both runs.

No data of the non-successful sessions were used for analysis.

\subsubsection{Stimulus material}

The stimulus set comprised 180 coloured images of faces and bodies of dogs and humans, everyday inanimate objects (e.g., a toy, a chair), and phase-grid scrambled versions of each category (30 images per condition) derived from Wagner and colleagues [96], the Hemera Photo-Object data base (Hemera Technologies) and the internet (see Figure 1B for examples). The images were resized to $600 \times 600$ pixels and presented in the centre of the MR screen on grey background. We cut out the heads off from the body images, as well as objects from body images (e.g., a coffee cup, a soccer ball). The images showed a variety of postures (e.g., jumping, looking

367 up), neutral and positive emotional displays (e.g., sleeping, smiling) and viewing 368 perspectives (e.g., from above, from a side angle). Luminance was set homogeneously 369 across stimuli and we controlled for spatial extent (see Supplementary file 2A for 370 details).

\section{$371 \quad 4.3$ MRI data acquisition}


Neural bases of face and body perception in dogs and humans

We acquired all MRI data with a 3T Siemens Skyra MR-system (Siemens

373 Medical, Erlangen, Germany) and a 15-channel coil (initially designed for

374 measurements of the human knee) for data acquisition in dogs and a 32-channel

375 human head coil for data acquisition in humans. Functional scans of dogs used a 2-

376 fold multiband (MB) accelerated echo planar imaging (EPI) sequence including the

377 following parameters: voxel size $=1.5 \times 1.5 \times 2 \mathrm{~mm}^{3}$, repetition time (TR) / echo time

$378($ TE $)=1000 / 38 \mathrm{~ms}$, field of view $($ FoV $)=144 \times 144 \times 58 \mathrm{~mm}^{3}$, flip angle $=61^{\circ}, 20 \%$

379 gap and 24 axial slices covering the whole brain (interleaved acquisition, descending

380 order). Structural scans had a voxel size of $0.7 \mathrm{~mm}$ isotropic (TR/TE $=2100 / 3.13 \mathrm{~ms}$,

381 FoV $=230 \times 230 \times 165 \mathrm{~mm}^{3}$ ) and were acquired in a separate scan session prior to

382 functional data collection. Human functional scans were acquired using a 4-fold MB

383 accelerated EPI sequence including the following parameters: voxel size $=2 \mathrm{~mm}$

384 isotropic, TR/TE $=1200 / 34 \mathrm{~ms}, \mathrm{FoV}=192 \times 192 \times 124.8 \mathrm{~mm}^{3}$, flip angle $=66^{\circ}, 20 \%$

385 gap and 52 axial slices coplanar to the connecting line between anterior and posterior

386 commissure (interleaved acquisition, ascending order). Additionally, we obtained field

387 map scans to correct functional scans for magnetic field inhomogeneities using a

388 double echo gradient echo sequence with the following parameters: voxel size $=1.72$

$389 \times 1.72 \times 3.85 \mathrm{~mm}^{3}$, TR/TE1/TE2 $=400 / 4.92 / 7.38 \mathrm{~ms}$, FoV $=220 \times 220 \times 138 \mathrm{~mm}^{3}$, flip

390 angle $=60^{\circ}$ and 36 axial slices (same orientation as functional scans). Structural scans

391 had a voxel size of $0.8 \mathrm{~mm}$ isotropic (TR/TE $=2300 / 2.43 \mathrm{~ms}$,

392 FoV $=256 \times 256 \times 166 \mathrm{~mm}^{3}$ ) and were acquired after functional data acquisition.

$393 \quad 4.4$ Data processing and statistical analysis

394 Imaging data was preprocessed and analyzed using SPM12

395 (https://www.fil.ion.ucl.ac.uk/spm/software/spm12/), Matlab 2018b (MathWorks) and R $396 \quad 3.6 .3[97]$. 
Neural bases of face and body perception in dogs and humans

\subsubsection{MRI data preprocessing}

In both samples, we slice-time corrected (reference: middle slice) and realigned functional images to the mean image. Human imaging data was also unwarped using the acquired field map. Dog imaging data was manually reoriented with the rostral commissure set as a visual reference (SPM module: "Reorient images / Set origin") to match the template orientation [51] and structural images were skull-stripped using individual binary brain masks created using itk-SNAP [98]. Next, we co-registered the structural and functional images to the mean functional image, segmented the structural images in both samples and normalized the human data to the Montreal Neurological Institute (MNI) template space and the dog data to a breed-averaged stereotaxic template space [51]. Normalized images were resliced to $1.5 \mathrm{~mm}$ isotropic and smoothed with a 3-dimensional Gaussian kernel (full-width-at-half-maximum, FWHM; with twice the raw voxel resolution: dogs/humans $=3 / 4 \mathrm{~mm}$; see [49] for an indepth description of our dog data preprocessing pipeline). We then calculated individual scan-to-scan motion (frame wise displacement, FD) and added motion regressors to first-level GLMs for each scan exceeding the a priori set FD threshold of $0.5 \mathrm{~mm}$ (motion scrubbing $[99,100]$ ) to account for both translational and rotational displacements. For the dog participants, we removed on average $8 \%$ of the scans from each run (run 1: mean $\mathrm{FD}=0.23 \mathrm{~mm}, 90^{\text {th }}$ percentile $=0.36 \mathrm{~mm}$; run 2: mean $\mathrm{FD}=$ $0.24 \mathrm{~mm}, 90^{\text {th }}$ percentile $=0.38 \mathrm{~mm}$ ) and $1 \%$ of the scans from each run of the human participants (run 1: mean FD $=0.17 \mathrm{~mm}, 90^{\text {th }}$ percentile $=0.22 \mathrm{~mm}$; run 2: mean $\mathrm{FD}=$ $0.18 \mathrm{~mm}, 90^{\text {th }}$ percentile $=0.21 \mathrm{~mm}$ ).

\subsection{2 fMRI data analysis}

Mass-univariate activation analysis. We analysed the functional data using a general linear model (GLM) approach implemented in SPM12. Individual GLM matrices included six regressors of interest for task (dog faces, dog bodies, human 
Neural bases of face and body perception in dogs and humans

423 faces, human bodies, inanimate objects, scrambled) and the six regressors from the

424 realignment procedure (see above) were added as nuisance regressors. All blocks

425 were estimated using a boxcar function time-locked to the onset of each block with a

426 duration of $12 \mathrm{~s}$. For the dog data, the signal was convolved with a tailored dog

427 haemodynamic response function [49] (HRF), while the standard human canonical

428 HRF (i.e., the default HRF parameters provided by SPM12) was used for the human

429 data. The dog HRF reflects a previously observed 2-3 s earlier peak of the BOLD signal

430 than expected by the human HRF model [49]. Normalized, individual binary masks

431 served as explicit masks and we applied a high-pass filter with a cut-off at $128 \mathrm{~s}$. We

432 then split the data in two sets (task run 1, task run 2). Based on the data from the first

433 task run, we estimated three subject-level contrast maps for the difference between

434 our conditions of interest (i.e., faces, bodies with equal weights for human and dog

435 images) and objects, and a visual stimulation contrast (all conditions $>$ implicit visual

436 baseline). For the second task run, we computed eight subject-level contrasts, one for

437 each task regressor (i.e., dog bodies, human bodies, dog faces, human faces,

438 everyday inanimate objects, scrambled), averaged for faces (i.e., dog and human

439 faces) and averaged for bodies (i.e., dog and human bodies) compared to implicit

440 visual baseline.

441 Functional region of interest (fROI) approach. We implemented a standard

442 functional region-of-interest (fROI, [54-56]) approach to investigate potential category-

443 specificity of cortical regions. This approach allows us to directly test the potential

444 category-sensitivity quantified by increased activation level while accounting for slight

445 differences in the location of activation peaks between individuals as which have been

446 reported in previous dog imaging studies [41,43].

447 The participant-level contrast images from the first run served as localizer data to 448 define individual category-sensitive regions. Within anatomically constrained search 
Neural bases of face and body perception in dogs and humans

spaces (Figure 2A) we selected the top-10\% voxel from each hemisphere with the

strongest signal for the condition-of-interest (i.e., face areas: faces > objects, body areas: bodies > objects) to form bilateral individual fROIs. Selecting the top-10\% voxel was an a priori decision we made because the resulting fROI sizes (on average: 30 voxels) were similar to the average cluster size observed in a previous dog imaging study that used two visual paradigms and the same smoothing kernel [49]. Further, percentages instead of absolute numbers took also the varying search space sizes into account (see Supplementary file 2B and Figure 2 - figure supplements 1-2 for more information). The data from the left out second run allowed then to directly test potential category specificity in an independent data set. Thus, we extracted parameter estimates from the conditions-of-interest contrasted against implicit visual baseline from both body and faces areas (= individual fROIs) using the REX toolbox [101]. analyses of variance (ANOVAs). First, we tested our main research question, whether

463 the body areas resulted in increased sensitivity for bodies regardless of species and 464 vice versa for faces, and added everyday objects and scrambled images as further conditions. Thus, we ran a one-way ANOVA with stimuli category (faces, bodies, everyday objects, scrambled) as dependent variable. Next, to investigate if there is a

467 difference in activation between conspecific and heterospecific stimuli, we used $2 \times 2$ 468 within-subjects ANOVAs (species: conspecific, heterospecific; image category: face, body). P-values for group comparisons investigating the same research questions

470 were Bonferroni-adjusted, e.g., if the 6 potential body fROls result in increased 471 sensitivity for bodies compared to all other categories results in an adjusted $p$-value of $4720.008(=0.05 / 6)$. Adjusted $p$-values are reported along with the results 473 (Supplementary File 1: Tables S2-3). For whole-brain group analyses, we tested 474 significance applying a whole-brain familywise error (FWE) correction for the human 
Neural bases of face and body perception in dogs and humans

data and a cluster-level inference with a cluster defining threshold of $p<0.001$ and a

cluster probability of $p<0.05$ FWE corrected for multiple comparisons for the dog data.

477 Cluster extent (i.e., minimum spatial extend to be labelled significant) was calculated

478 using the SPM extension "CorrClusTh.m" (by Thomas Nichols, University of Warwick,

479 United Kingdom, and Marko Wilke, University of Tübingen, Germany;

https://warwick.ac.uk/fac/sci/statistics/staff/academic-research/nichols/scripts/spm/).

Representational similarity analysis. Next, we investigated the neural

482 representations for faces, bodies and objects and their potential convergence in dogs

483 and humans. To this end, we performed a whole-brain representational similarity 484 analysis (RSA; [61-63]) to determine neural pattern similarities within image categories. GLMs were modelled identical to univariate GLMs (see above) but for each

block, we ran a separate GLM with the block as task regressor and remaining blocks were combined in one regressor of no interest [102]; runs were modelled independently. Thus, the analysis resulted in 36 beta images for each participant (6 conditions $\times 6$ trials). RSA was performed using the smoothed functional data. For all RSA analyses, we moved a spherical searchlight [103] (dogs: $r=4 \mathrm{~mm}, 81$ voxel;

491 humans: $r=8 \mathrm{~mm}, 251$ voxel) throughout individual whole-brain grey matter masks computed based on the normalised segmentation output considering only searchlights with a minimum of 15 grey matter voxel for the dog and 30 for the human data.

Within a given searchlight, we extracted single-trial beta estimates from all voxels

and sorted them according to their stimuli category (Figure 3A; dog / human bodies, dog / human faces, inanimate objects) and reshaped the data to a trial $\times$ voxel matrix.

497 Next, we $z$-scored the data across trials and runs and computed a trial $\times$ trial similarity 498 matrix by correlating each beta estimate of each trial to all other trial estimates. Finally, 499 in order to retrieve overall similarity scores, we then Fisher's z-transformed the data and calculated overall similarity matrices by averaging scores across the respective 
Neural bases of face and body perception in dogs and humans

501 quadrants. We were specifically interested in pattern similarities across animate vs.

502 inanimate (faces $\times$ bodies vs. inanimate objects), faces or bodies vs. inanimate objects,

503 faces vs. bodies and conspecific vs. heterospecific species dimensions within face and

504 body categories. We then assigned the overall similarity values to the centre voxel of each searchlight resulting in individual whole-brain pattern similarity maps.

507 between trials of (1) faces vs. inanimate objects (i.e., [dog faces $\times$ human faces] vs. 508 inanimate objects), (2) bodies vs. inanimate objects (i.e., [dog bodies $\times$ human bodies] 509 vs. inanimate objects), (3) animate vs. inanimate images (i.e., [dog faces $\times$ human 510 faces $\mathrm{x}$ dog bodies $\times$ human bodies] vs. inanimate objects), (4) faces vs. bodies (i.e., 511 [dog faces $\times$ human faces] vs. [dog bodies $\times$ human bodies]), and $(5,6)$ within the face 512 and body categories: images of conspecifics vs. heterospecifics (i.e., dog faces vs. 513 human faces; dog bodies vs. human bodies). We computed permutation tests [104] to

514 determine group-level significance using the Statistical nonParametric Mapping 515 (SnPm13, http://www.nisox.org/Software/SnPM13/) toolbox running 5000 516 permutations for each paired $t$-test and applied cluster-level inference with a cluster 517 defining threshold of $p<.005 / .001$ (dogs/humans) and a cluster probability of $p<0.05$ 518 familywise error corrected (FWE) for multiple comparisons.

Data visualization.

Data was visualized using SPM12

520 (https://www.fil.ion.ucl.ac.uk/spm/software/spm12/), Matlab 2018b (MathWorks), itk521 SNAP [98], R 3.6.3 [97] mainly using the packages ggplot2 [105] and RainCloudPlots 522 [106] and the python project nilearn (http://nilearn.github.io).

\section{Data availability statement}

524 The code to create functional regions-of-interest (fROls) within anatomical 525 search spaces and to extract activations levels, as well as the representational 
Neural bases of face and body perception in dogs and humans

similarity analysis (RSA) code are openly available on Github

527 (https://github.com/magdalenaboch/fROI-analysis,

528 https://github.com/isabellawagner/searchlight-rsa). The extracted fROI activation

529 levels along with the $\mathrm{R}$ code to visualize and analyse the data, the overlap maps of

530 individual fROIs, thresholded and unthresholded statistical maps of univariate (all >

531 implicit baseline) and RSA analysis and averaged structural images to serve as

532 background images to overlay the data on have been uploaded to the Open Science

533 Framework and are openly available at https://osf.io/kzcs2/.

\section{Acknowledgments}

We thank Prof. Nancy Kanwisher for her helpful comments on the preliminary

537 Borghi and Helena Manzenreiter for their help collecting the data, Boryana Todorova

538 for her support in preparing the stimuli set and all the dogs and their caregivers and all

539 human participants for taking part in this project.

\section{CRediT authorship statement}

Magdalena Boch: Conceptualization, Methodology, Software, Validation,

542 Formal analysis, Investigation, Data curation, Writing - original draft, Writing - review \&

543 editing, Visualization, Project administration. Isabella C. Wagner: Conceptualization,

544 Methodology, Resources, Writing - original draft, Writing - review \& editing,

545 Supervision. Sabrina Karl: Investigation, Writing - review \& editing. Ludwig Huber:

546 Conceptualization, Resources, Writing - review \& editing, Supervision, Funding

547 acquisition. Claus Lamm: Conceptualization, Methodology, Resources, Writing -

548 original draft, Writing - review \& editing, Supervision, Funding acquisition. 


\section{Declaration of Competing Interests}

551 The authors declare no competing financial interests.

\section{Supplementary Files}

Supplementary file 1. Supplementary tables: Table S1. Task-related

554 activation: localizer data set (task run 1), Table S2. Results from one-way ${ }^{a}$ repeated

555 measures analyses of variance (ANOVAs), Table S3. Results from $2 \times 2^{\mathrm{a}}$ repeated

556 measures analyses of variance (ANOVAs), Table S4. Dog data: Pattern similarity

557 during face, body and object perception and Table S5. Human data: Pattern

558 similarity during face, body and object perception.

559 Supplementary file 2. 2A: Stimulus material: luminance and spatial extent, 2B:

560 Anatomical search spaces and size individual fROls 
bioRxiv preprint doi: https://doi org/10.1101/2021.08.17.456623; this version posted September 20, 2021. The copyright holder for this preprint (which was not certified by peer review) is the author/funder, who has granted bioRxiv a license to display the preprint in perpetuity. It is made available under aCC-BY-NC-ND 4.0 International license.

Neural bases of face and body perception in dogs and humans

\title{
10 Figure supplements
}

A Caudal suprasylvian gyrus
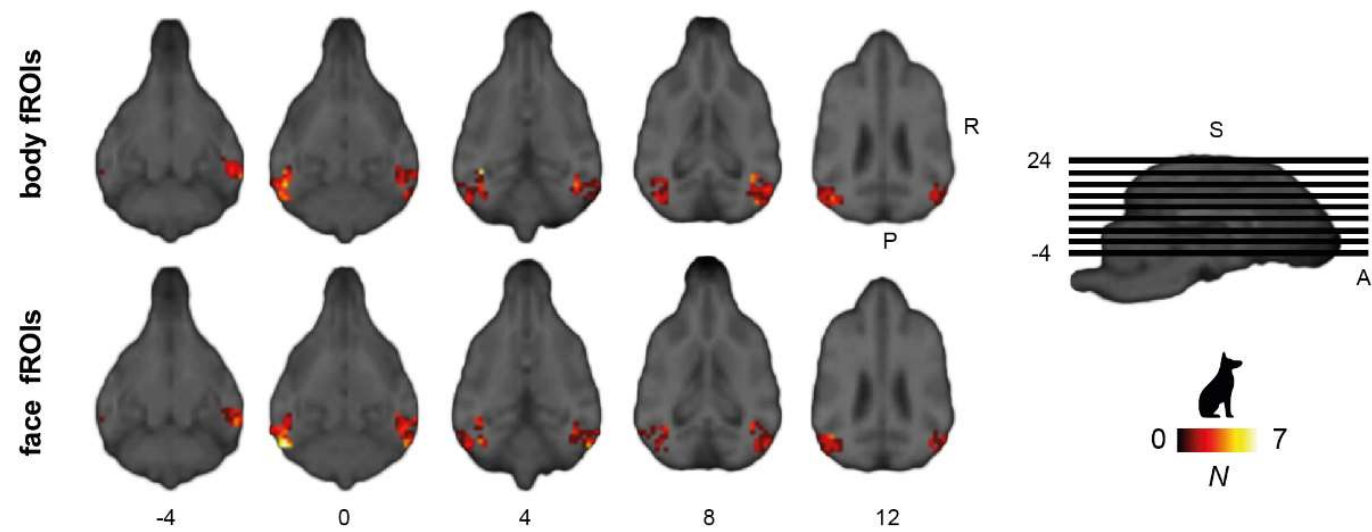

B Medial suprasylvian gyrus

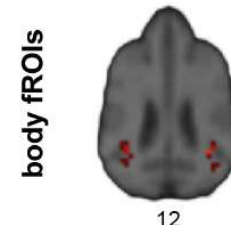

12

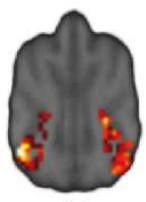

16

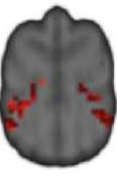

20

12

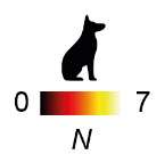

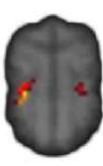

24

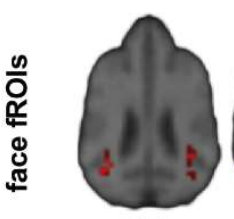

12

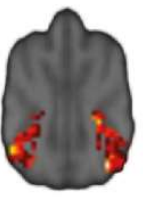

16

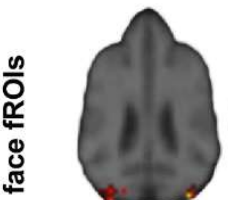

12

24

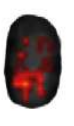

28

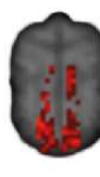

24

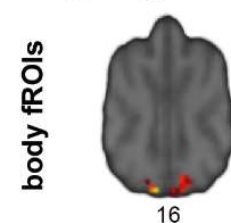

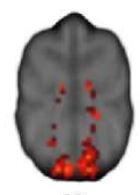

20

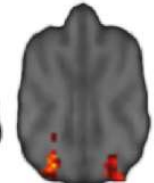

16

(200

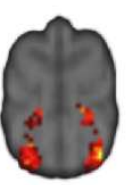

20

28

\begin{abstract}
Figure 2 - figure supplement 1. Overlap of individual functional regions-of-interest (fRO/s) from dog participants. Individual fROls were defined for faces (> inanimate objects) and bodies (> inanimate objects) within multiple anatomical search spaces (A-D) based on the data from localizer data set (i.e., first task run). The colour of the heat map indicates the amount of overlap between the fROls of all dog participants. We observed slight individual differences in anatomical location between participants as indicated by a maximum overlap of $N=7$ from overall 15 participants. The axial plane in panel A (top row) shows the anatomical locations posterior $(P)$ and right hemisphere $(R)$; all axial planes displayed have the same orientation. The sagittal plane displays the cut coordinates $(\mathrm{z})$ and the anatomical locations superior $(\mathrm{S})$ and anterior $(\mathrm{A})$; z-coordinates refer to a canine-breed averaged template [51].
\end{abstract}


bioRxiv preprint doi: https://doi org/101101/2021.08.17.456623; this version posted September 20, 2021. The copyright holder for this preprint (which was not certified by peer review) is the author/funder, who has granted bioRxiv a license to display the preprint in perpetuity. It is made available under aCC-BY-NC-ND 4.0 International license.

Neural bases of face and body perception in dogs and humans

A Extrastriate body area (body fROI)

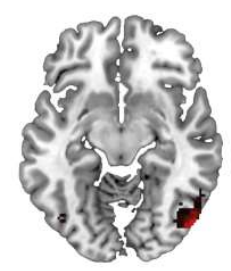

$-10$

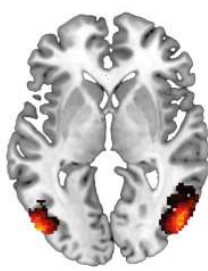

0

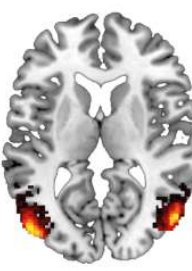

10

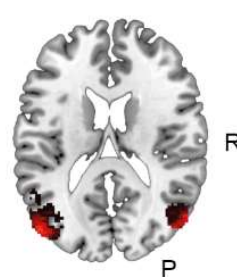

20

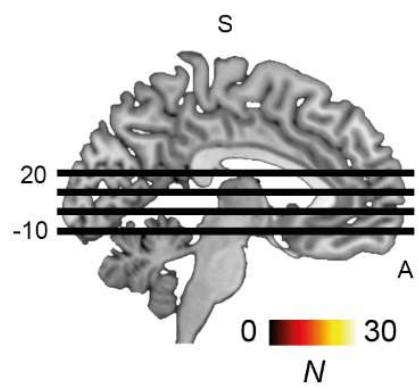

B Fusiform body area (body fROI)

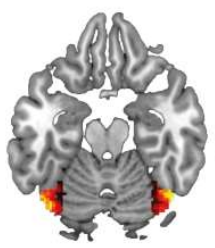

$-20$

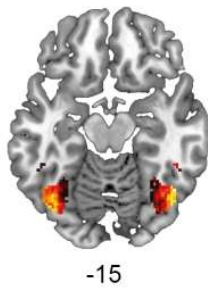

$-15$

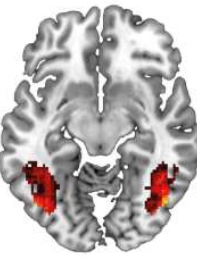

$-10$

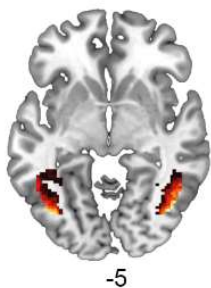

C Occipital face area (face fROI)

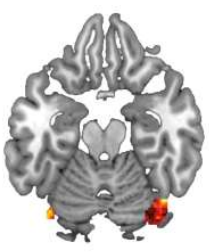

$-20$

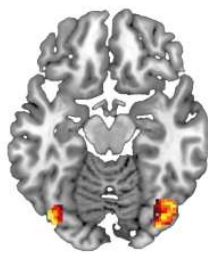

$-15$

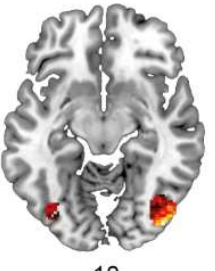

$-10$

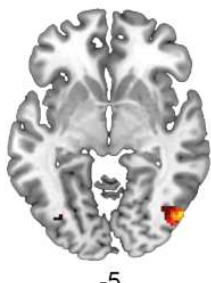

$-5$

D Fusiform face area (face fROI)

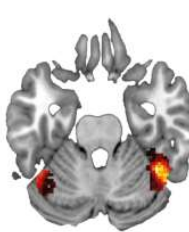

$-25$

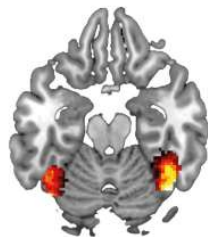

$-20$

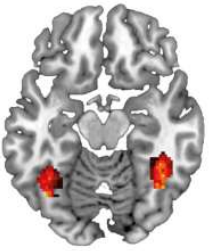

$-15$

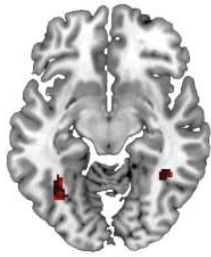

$-10$
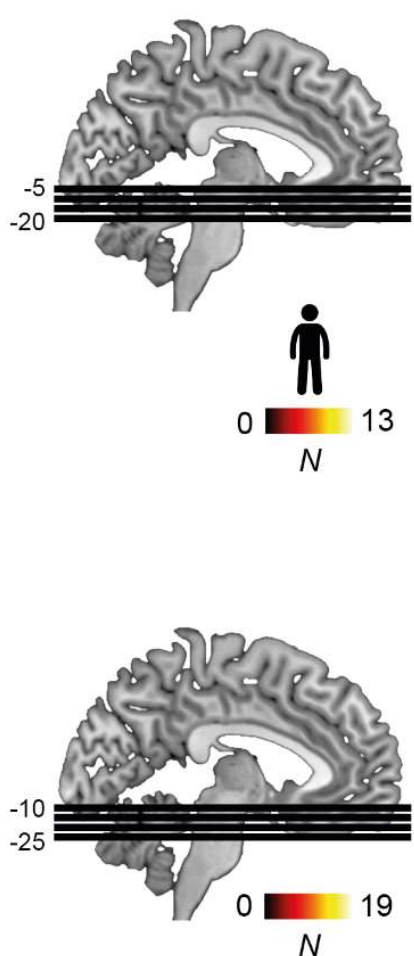

Figure 2 - figure supplement 2. Overlap of individual functional regions-of-interest (fROIs) from human participants. Individual fROIs were defined for faces ( $>$ inanimate objects) and bodies ( $>$ inanimate objects) within multiple anatomical search spaces (A-D) based on the data from the localizer data set (i.e. first task run). The colour of the heat map indicates the amount of overlap between the fROls of all humans. We observed slight individual differences in anatomical location between participants as indicated by a maximum overlap of $N=30$ from overall 40 participants. The axial plane in panel A shows the anatomical locations posterior $(P)$ and right hemisphere $(R)$; all axial planes displayed have the same orientation. The sagittal planes display the cut coordinates $(\mathrm{z})$ and the anatomical locations superior ( $\mathrm{S}$ ) and anterior. 
Neural bases of face and body perception in dogs and humans

\section{References}

566 1. Rajhans P, Jessen S, Missana M, Grossmann T. Putting the face in context: Body expressions impact facial emotion processing in human infants. Dev Cogn Neurosci. 2016;19: 115-121. doi:10.1016/J.DCN.2016.01.004

2. Aviezer $\mathrm{H}$, Trope $\mathrm{Y}$, Todorov A. Body cues, not facial expressions, discriminate

3. Barrett LF, Mesquita B, Gendron M. Context in emotion perception. Curr Dir between intense positive and negative emotions. Science. 2012;338: 1225-

4. Lecker M, Dotsch R, Bijlstra G, Aviezer H. Bidirectional contextual influence between faces and bodies in emotion perception. Emotion. 2020;20: 11541164. doi:10.1037/EMO0000619

5. Poyo Solanas M, Zhan M, Vaessen M, Hortensius R, Engelen T, de Gelder B. Looking at the face and seeing the whole body. Neural basis of combined face and body expressions. Soc Cogn Affect Neurosci. 2018;13: 135-144.

582

6. de Gelder B, de Borst AW, Watson R. The perception of emotion in body expressions. Wiley Interdiscip Rev Cogn Sci. 2015;6: 149-158. doi:10.1002/wcs.1335

7. Müller CA, Schmitt K, Barber ALA, Huber L. Dogs can discriminate emotional expressions of human faces. Curr Biol. 2015;25: 601-605. doi:10.1016/j.cub.2014.12.055

8. Kravitz DJ, Saleem KS, Baker CI, Ungerleider LG, Mishkin M. The ventral visual pathway: An expanded neural framework for the processing of object quality. Trends Cogn Sci. 2013;17: 26-49. doi:10.1016/j.tics.2012.10.011

9. Ungerleider LG, Haxby J V. "What" and "where" in the human brain. Curr Opin Neurobiol. 1994;4: 157-165. doi:10.1016/0959-4388(94)90066-3

10. Schwarzlose RF, Baker $\mathrm{Cl}$, Kanwisher N. Separate face and body selectivity on the fusiform gyrus. J Neurosci. 2005;25: 11055-11059. doi:10.1523/JNEUROSCI.2621-05.2005

11. Peelen M V., Downing PE. Selectivity for the human body in the fusiform gyrus. J Neurophysiol. 2005;93: 603-608. doi:10.1152/jn.00513.2004 
13. Downing PE, Jiang $\mathrm{Y}$, Shuman M, Kanwisher N. A cortical area selective for visual processing of the human body. Science. 2001;293: 2470-2473. doi:10.1126/science.1063414

14. Spiridon M, Fischl B, Kanwisher N. Location and spatial profile of categoryspecific regions in human extrastriate cortex. Hum Brain Mapp. 2006;27: 7789. doi:10.1002/hbm.20169

15. Kanwisher N. Domain specificity in face perception. Nat Neurosci. 2000;3: 759-763. doi:10.1038/77664

16. Yovel G, Kanwisher N. Face perception: domain specific, not process specific. Neuron. 2004;44: 889-898. doi:10.1016/j.neuron.2004.11.018

17. Wegrzyn M, Riehle M, Labudda K, Woermann F, Baumgartner F, Pollmann S, et al. Investigating the brain basis of facial expression perception using multivoxel pattern analysis. Cortex. 2015;69: 131-140. doi:10.1016/J.CORTEX.2015.05.003

18. Wiggett AJ, Pritchard IC, Downing PE. Animate and inanimate objects in human visual cortex: Evidence for task-independent category effects. Neuropsychologia. 2009;47: 3111-3117. doi:10.1016/J.NEUROPSYCHOLOGIA.2009.07.008

19. Chao LL, Haxby J V., Martin A. Attribute-based neural substrates in temporal

20. Downing PE, Chan AW-Y, Peelen M V., Dodds CM, Kanwisher N. Domain specificity in visual cortex. Cereb Cortex. 2006;16: 1453-1461. doi:10.1093/CERCOR/BHJ086

21. Tsao DY, Freiwald WA, Knutsen TA, Mandeville JB, Tootell RBH. Faces and objects in macaque cerebral cortex. Nat Neurosci. 2003;6: 989-995. doi:10.1038/nn1111

22. Desimone R, Albright TD, Gross CG, Bruce C. Stimulus-selective properties of inferior temporal neurons in the macaque. J Neurosci. 1984;4: 2051-2062. doi:10.1523/jneurosci.04-08-02051.1984

23. Wachsmuth E, Oram MW, Perrett DI. Recognition of objects and their component parts: Responses of single units in the temporal cortex of the macaque. Cereb Cortex. 1994;4: 509-522. doi:10.1093/cercor/4.5.509

24. Gross CG, Bender DB, Rocha-Miranda CE. Visual receptive fields of neurons in inferotemporal cortex of the monkey. Science. 1969;166: 1303-1306. 
Neural bases of face and body perception in dogs and humans doi:10.1126/science.166.3910.1303

25. Haxby J V., Horwitz B, Ungerleider LG, Maisog JM, Pietrini P, Grady CL. The functional organization of human extrastriate cortex: a PET-rCBF study of selective attention to faces and locations. J Neurosci. 1994;14: 6336-6353. doi:10.1523/JNEUROSCI.14-11-06336.1994

26. Kanwisher N, Yovel G. The fusiform face area: a cortical region specialized for the perception of faces. Philos Trans R Soc B Biol Sci. 2006;361: 2109-2128. doi:10.1098/rstb.2006.1934

27. Tsao DY, Freiwald WA, Tootell RBH, Livingstone MS. A cortical region consisting entirely of face-selective cells. Science. 2006;311: 670-674. doi:10.1126/science.1119983

28. Tsao DY, Moeller S, Freiwald WA. Comparing face patch systems in macaques and humans. Proc Natl Acad Sci U S A. 2008;105: 19514-19519. doi:10.1073/pnas.0809662105

29. Bunford N, Hernández-Pérez R, Farkas EB, Cuaya L V., Szabó D, Szabó ÁG, et al. Comparative Brain Imaging Reveals Analogous and Divergent Patterns of Species and Face Sensitivity in Humans and Dogs. J Neurosci. 2020;40: 8396-8408. doi:10.1523/JNEUROSCI.2800-19.2020

30. Kanwisher N, Stanley D, Harris A. The fusiform face area is selective for faces not animals. Neuroreport. 1999;10: 183-187. doi:10.1097/00001756199901180-00035

31. Pinsk MA, Arcaro M, Weiner KS, Kalkus JF, Inati SJ, Gross CG, et al. Neural representations of faces and body parts in macaque and human cortex: a comparative fMRI study. J Neurophysiol. 2009;101: 2581-2600. doi:10.1152/jn.91198.2008

32. Hesse JK, Tsao DY. The macaque face patch system: a turtle's underbelly for the brain. Nat Rev Neurosci 2020 2112. 2020;21: 695-716.

doi:10.1038/s41583-020-00393-w

33. Racca A, Amadei E, Ligout S, Guo K, Meints K, Mills D. Discrimination of human and dog faces and inversion responses in domestic dogs (Canis familiaris). Anim Cogn. 2010;13: 525-533. doi:10.1007/s10071-009-0303-3

34. Huber L, Racca A, Scaf B, Virányi Z, Range F. Discrimination of familiar human faces in dogs (Canis familiaris). Learn Motiv. 2013;44: 258-269. doi:10.1016/J.LMOT.2013.04.005 
Neural bases of face and body perception in dogs and humans

dog and human emotions. Biol Lett. 2016;12: 20150883.

doi:10.1098/rsbl.2015.0883

36. Huber L. How Dogs Perceive and Understand Us. Curr Dir Psychol Sci. 2016;25: 339-344. doi:10.1177/0963721416656329

37. Maginnity ME, Grace RC. Visual perspective taking by dogs (Canis familiaris) in a Guesser-Knower task: evidence for a canine theory of mind? Anim Cogn. 2014;17: 1375-1392. doi:10.1007/s10071-014-0773-9

38. Catala A, Mang B, Wallis L, Huber L. Dogs demonstrate perspective taking based on geometrical gaze following in a Guesser-Knower task. Anim Cogn. 2017;20: 581-589. doi:10.1007/s10071-017-1082-x

39. Bunford N, Andics A, Kis A, Miklósi Á, Gácsi M. Canis familiaris As a Model for Non-Invasive Comparative Neuroscience. Trends Neurosci. 2017;40: 438-452. doi:10.1016/J.TINS.2017.05.003

40. Szabó D, Gábor A, Gácsi M, Faragó T, Kubinyi E, Miklósi Á, et al. On the Face of It: No Differential Sensitivity to Internal Facial Features in the Dog Brain. Front Behav Neurosci. 2020;14. doi:10.3389/fnbeh.2020.00025

41. Dilks DD, Cook PF, Weiller SK, Berns HP, Spivak M, Berns GS. Awake fMRI reveals a specialized region in dog temporal cortex for face processing. PeerJ. 2015; 3:e1115. doi:10.7717/peerj.1115

42. Thompkins AM, Ramaiahgari B, Zhao S, Gotoor SSR, Waggoner P, Denney TS, et al. Separate brain areas for processing human and dog faces as revealed by awake $\mathrm{fMRI}$ in dogs (Canis familiaris). Learn Behav. 2018;46: 561-573. doi:10.3758/s13420-018-0352-z

43. Cuaya L V., Hernández-Pérez R, Concha L. Our faces in the dog's brain: Functional imaging reveals temporal cortex activation during perception of human faces. PLoS One. 2016;11: e0149431. doi:10.1371/journal.pone.0149431

44. Siniscalchi M, Lusito R, Vallortigara G, Quaranta A. Seeing Left- or RightAsymmetric Tail Wagging Produces Different Emotional Responses in Dogs. Curr Biol. 2013;23: 2279-2282. doi:10.1016/J.CUB.2013.09.027

45. Duranton C, Range F, Virányi Z. Do pet dogs (Canis familiaris) follow ostensive and non-ostensive human gaze to distant space and to objects? R Soc Open Sci. 2017;4: 170349. doi:10.1098/rsos.170349

46. Topál J, Kis A, Oláh K. Dogs' Sensitivity to Human Ostensive Cues: A Unique Adaptation? The Social Dog: Behavior and Cognition. Elsevier Inc.; 2014. pp. 

319-346. doi:10.1016/B978-0-12-407818-5.00011-5

47. Bräuer J, Kaminski J, Riedel J, Call J, Tomasello M. Making inferences about the location of hidden food: Social dog, causal ape. J Comp Psychol. 2006;120: 38-47. doi:10.1037/0735-7036.120.1.38

48. Fitch WT, Huber L, Bugnyar T. Social Cognition and the Evolution of Language: Constructing Cognitive Phylogenies. Neuron. 2010;65: 795-814. doi:10.1016/J.NEURON.2010.03.011

49. Boch M, Karl S, Sladky R, Huber L, Lamm C, Wagner IC. Tailored haemodynamic response function increases detection power of $\mathrm{fMRI}$ in awake dogs (Canis familiaris). Neuroimage. 2021;224: 117414. doi:10.1016/j.neuroimage.2020.117414

50. Karl S, Boch M, Virányi Z, Lamm C, Huber L. Training pet dogs for eye-tracking and awake fMRI. Behav Res Methods. 2020;52. doi:10.3758/s13428-01901281-7

51. Nitzsche B, Boltze J, Ludewig E, Flegel T, Schmidt MJ, Seeger J, et al. A

52. Czeibert K, Andics A, Petneházy Ö, Kubinyi E. A detailed canine brain label map for neuroimaging analysis. Biol Futur. 2019;70: 112-120. doi:10.1556/019.70.2019.14

53. Shattuck DW, Mirza M, Adisetiyo V, Hojatkashani C, Salamon G, Narr KL, et al. Construction of a 3D probabilistic atlas of human cortical structures. Neuroimage. 2008;39: 1064-1080. doi:10.1016/j.neuroimage.2007.09.031

55. Poldrack RA. Region of interest analysis for fMRI. Soc Cogn Affect Neurosci. 2007;2: 67-70. doi:10.1093/scan/nsm006

56. Saxe R, Brett M, Kanwisher N. Divide and conquer: A defense of functional localizers. Neuroimage. 2006;30: 1088-1096. doi:10.1016/J.NEUROIMAGE.2005.12.062

737 57. Julian JB, Fedorenko E, Webster J, Kanwisher N. An algorithmic method for functionally defining regions of interest in the ventral visual pathway. 
Neuroimage. 2012;60: 2357-2364. doi:10.1016/j.neuroimage.2012.02.055

58. Karl S, Boch M, Zamansky A, van der Linden D, Wagner IC, Völter CJ, et al. Exploring the dog-human relationship by combining $\mathrm{fMRI}$, eye-tracking and behavioural measures. Sci Rep. 2020;10: 22273. doi:10.1038/s41598-02079247-5

59. Thompkins AM, Lazarowski L, Ramaiahgari B, Gotoor SSR, Waggoner P, Denney TS, et al. Dog-human social relationship: representation of human face familiarity and emotions in the dog brain. Anim Cogn. 2021;24: 251-266. doi:10.1007/s10071-021-01475-7

60. Cohen MA, Dilks DD, Koldewyn K, Weigelt S, Feather J, Kell AJE, et al.

Representational similarity precedes category selectivity in the developing ventral visual pathway. Neuroimage. 2019;197: 565-574. doi:10.1016/j.neuroimage.2019.05.010

61. Kriegeskorte N, Kievit RA. Representational geometry: integrating cognition, computation, and the brain. Trends Cogn Sci. 2013;17: 401-412. doi:10.1016/J.TICS.2013.06.007

62. Kriegeskorte N, Mur M, Bandettini P. Representational similarity analysis connecting the branches of systems neuroscience. Front Syst Neurosci. 2008;2. doi:10.3389/neuro.06.004.2008

63. Kriegeskorte N, Mur M, Ruff DA, Kiani R, Bodurka J, Esteky H, et al. Matching Categorical Object Representations in Inferior Temporal Cortex of Man and Monkey. Neuron. 2008;60: 1126-1141. doi:10.1016/J.NEURON.2008.10.043

64. Rizzolatti G, Fogassi L, Gallese V. Neurophysiological mechanisms underlying the understanding and imitation of action. Nat Rev Neurosci. 2001;2: 661-670. doi:10.1038/35090060

65. Hamilton AF de C, Grafton ST. Goal Representation in Human Anterior Intraparietal Sulcus. J Neurosci. 2006;26: 1133-1137. doi:10.1523/JNEUROSCI.4551-05.2006

66. Kilner JM, Neal A, Weiskopf N, Friston KJ, Frith CD. Evidence of Mirror

67. Törnqvist $\mathrm{H}$, Somppi S, Kujala M V., Vainio O. Observing animals and humans: Neurons in Human Inferior Frontal Gyrus. J Neurosci. 2009;29: 10153-10159. Dogs target their gaze to the biological information in natural scenes. PeerJ. 2020;8: e10341. doi:10.7717/peerj.10341 
Neural bases of face and body perception in dogs and humans

Comparison of dogs and humans in visual scanning of social interaction. R Soc Open Sci. 2015;2: 150341. doi:10.1098/rsos.150341

69. Spriet C, Abassi E, Hochmann J-R, Papeo L. Visual object categorization in infancy. bioRxiv. 2021; 2021.02.25.432436. doi:10.1101/2021.02.25.432436

70. Buttelmann D, Tomasello M. Can domestic dogs (Canis familiaris) use referential emotional expressions to locate hidden food? Anim Cogn. 2013. doi:10.1007/s10071-012-0560-4

71. Nagasawa M, Murai K, Mogi K, Kikusui T. Dogs can discriminate human smiling faces from blank expressions. Anim Cogn. 2011;14: 525-533. doi:10.1007/s10071-011-0386-5

72. Barber ALA, Randi D, Müller CA, Huber L. The Processing of Human Emotional Faces by Pet and Lab Dogs: Evidence for Lateralization and Experience Effects. Guo K, editor. PLoS One. 2016;11: e0152393. doi:10.1371/journal.pone.0152393

73. Racca A, Guo K, Meints K, Mills DS. Reading Faces: Differential Lateral Gaze Bias in Processing Canine and Human Facial Expressions in Dogs and 4-YearOld Children. PLoS One. 2012;7: e36076. doi:10.1371/journal.pone.0036076

74. Range F, Viranyi Z, Huber L. Selective Imitation in Domestic Dogs. Curr Biol. 2007;17: 868-872. doi:10.1016/J.CUB.2007.04.026

75. Soproni K, Miklósi Á, Topál J, Csányi V. Dogs' (Canis familiaris)

Responsiveness to Human Pointing Gestures. J Comp Psychol. 2002;116: 2734. doi:10.1037//0735-7036.116.1.27

76. Bray EE, Gnanadesikan GE, Horschler DJ, Levy KM, Kennedy BS, Famula TR, et al. Early-emerging and highly heritable sensitivity to human communication in dogs. Curr Biol. 2021;31: 1-5. doi:10.1016/j.cub.2021.04.055

77. Karl S, Sladky R, Lamm C, Huber L. Neural Responses of Pet Dogs Witnessing their caregiver's Positive Interactions with a Conspecific: An fMRI Study. Cereb Cortex Commun. 2021;tgab047. doi:10.1093/TEXCOM/TGAB047

78. Correia-Caeiro C, Guo K, Mills D. Bodily emotional expressions are a primary source of information for dogs, but not for humans. Anim Cogn. 2021;1: 3. doi:10.1007/s10071-021-01471-x

79. Salomons H, Smith KCM, Callahan-Beckel M, Callahan M, Levy K, Kennedy BS, et al. Cooperative Communication with Humans Evolved to Emerge Early in Domestic Dogs. Curr Biol. 2021;31: 3137-3144.e11. 
80. Kaminski J. Domestic dogs: Born human whisperers. Curr Biol. 2021;31: R891-R893. doi:10.1016/J.CUB.2021.05.063

81. Siniscalchi M, D'Ingeo S, Minunno M, Quaranta A. Communication in Dogs. Animals. MDPI AG; 2018. p. 131. doi:10.3390/ani8080131

82. Long RA, Donovan TM, Mackay P, Zielinski WJ, Buzas JS. Effectiveness of Scat Detection Dogs for Detecting Forest Carnivores. J Wildl Manage. 2007;71: 2007-2017. doi:10.2193/2006-230

83. Jamieson LTJ, Baxter GS, Murray PJ. Identifying suitable detection dogs. Applied Animal Behaviour Science. Elsevier B.V.; 2017. pp. 1-7. doi:10.1016/j.applanim.2017.06.010

84. Grimm-Seyfarth A, Harms W, Berger A. Detection dogs in nature conservation: A database on their world-wide deployment with a review on breeds used and their performance compared to other methods. Methods in Ecology and Evolution. British Ecological Society; 2021. pp. 568-579. doi:10.1111/2041210X.13560

85. Dunn M, Degenhardt L. The use of drug detection dogs in Sydney, Australia. Drug Alcohol Rev. 2009;28: 658-662. doi:10.1111/j.1465-3362.2009.00065.x

86. Guest C, Otto CM. Editorial: Canine Olfactory Detection. Frontiers in Veterinary

88. Prichard A, Chhibber R, Athanassiades K, Spivak M, Berns GS. Fast neural

87. Berns GS, Brooks AM, Spivak M. Scent of the familiar: An fMRI study of canine brain responses to familiar and unfamiliar human and dog odors. Behav Processes. 2015;110: 37-46. doi:10.1016/J.BEPROC.2014.02.011 learning in dogs: A multimodal sensory fMRI study. Sci Rep. 2018;8: 14614. doi:10.1038/s41598-018-32990-2

89. Jia H, Pustovyy OM, Wang Y, Waggoner P, Beyers RJ, Schumacher J, et al. Enhancement of Odor-Induced Activity in the Canine Brain by Zinc

90. Jia H, Pustovyy OM, Waggoner P, Beyers RJ, Schumacher J, Wildey C, et al. Nanoparticles: A Functional MRI Study in Fully Unrestrained Conscious Dogs.

91. Ramaingari B, Pustovyy OM, Waggoner P, Beyers RJ, Wildey C, Morrison E, et al. Zinc nanoparticles enhance brain connectivity in the canine olfactory network: Evidence from an fMRI study in unrestrained awake dogs. Front Vet 
Neural bases of face and body perception in dogs and humans

Sci. 2018;5: 127. doi:10.3389/fvets.2018.00127

92. Bräuer J, Blasi D. Dogs display owner-specific expectations based on olfaction. Sci Reports 2021 111. 2021;11: 1-10. doi:10.1038/s41598-021-82952-4

93. Hecht EE, Smaers JB, Dunn WJ, Kent M, Preuss TM, Gutman DA. Significant Neuroanatomical Variation Among Domestic Dog Breeds. J Neurosci. 2019;39: 0303-19. doi:10.1523/JNEUROSCI.0303-19.2019

94. Kilkenny C, Browne WJ, Cuthill IC, Emerson M, Altman DG. Improving

95. Peirce JW. PsychoPy-Psychophysics software in Python. J Neurosci Methods. 2007;162: 8-13. doi:10.1016/j.jneumeth.2006.11.017

96. Wagner IC, van Buuren M, Bovy L, Fernández G. Parallel engagement of regions associated with encoding and later retrieval forms durable memories. $J$ Neurosci. 2016;36: 7985-7995. doi:10.1523/JNEUROSCI.0830-16.2016

97. R Core Team. R: A Language and Environment for Statistical Computing. Vienna, Austria: R Foundation for Statistical Computing; 2020. Available: https://www.r-project.org/

98. Yushkevich PA, Piven J, Hazlett HC, Smith RG, Ho S, Gee JC, et al. Userguided 3D active contour segmentation of anatomical structures: Significantly improved efficiency and reliability. Neuroimage. 2006;31: 1116-1128. doi:10.1016/J.NEUROIMAGE.2006.01.015

99. Power JD, Barnes KA, Snyder AZ, Schlaggar BL, Petersen SE. Spurious but systematic correlations in functional connectivity MRI networks arise from subject motion. Neuroimage. 2012;59: 2142-2154. doi:10.1016/J.NEUROIMAGE.2011.10.018

100. Power JD, Mitra A, Laumann TO, Snyder AZ, Schlaggar BL, Petersen SE. Methods to detect, characterize, and remove motion artifact in resting state activation in event-related designs for multivoxel pattern classification analyses. Neuroimage. 2012;59: 2636-2643. 
103. Kriegeskorte N, Goebel R, Bandettini P. Information-based functional brain mapping. Proc Natl Acad Sci U S A. 2006;103: 3863-3868. doi:10.1073/pnas.0600244103

104. Nichols TE, Holmes AP. Non-parametric procedures. Statistical Parametric Mapping: The analysis of Functional Brain Images. Amsterdam: Elsevier; 2007. pp. 253-272.

105. Wickham H. ggplot2: Elegant Graphics for Data Analysis. Springer-Verlag New York; 2016. Available: https://ggplot2.tidyverse.org

106. Allen M, Poggiali D, Whitaker K, Marshall TR, Kievit RA. Raincloud plots: A multi-platform tool for robust data visualization [version 1; peer review: 2 approved]. Wellcome Open Res. 2019;4. doi:10.12688/wellcomeopenres.15191.1 and informative figures from statistical MRI images. J Open Source Softw. 